\begin{tabular}{|l|l|l||}
\hline \multicolumn{2}{|c|}{ PublisherInfo } \\
\hline \hline PublisherName & $:$ & BioMed Central \\
\hline \hline PublisherLocation & $:$ & London \\
\hline \hline PublisherImprintName & $:$ & BioMed Central \\
\hline \hline
\end{tabular}

\title{
Tamoxifen, angiogenesis, oestrogens and animal models
}

\begin{tabular}{|l|l|l||}
\hline \multicolumn{2}{|c|}{ ArticleInfo } \\
\hline \hline ArticleID & $:$ & 3758 \\
\hline \hline ArticleDOI & $:$ & $10.1186 /$ bcr-2001-66721 \\
\hline \hline ArticleCitationID & $:$ & 66721 \\
\hline \hline ArticleSequenceNumber & $:$ & 30 \\
\hline \hline ArticleCategory & $:$ & Paper Report \\
\hline ArticleFirstPage & $:$ & 1 \\
\hline \hline ArticleLastPage & $:$ & 4 \\
\hline \hline & & RegistrationDate : 2001-1-22 \\
\hline ArticleHistory & $:$ & OnlineDate \\
\hline \hline ArticleCopyright & $:$ & Biomed Central Ltd2001-1-22 \\
\hline \hline ArticleGrants & $:$ & \\
\hline \hline ArticleContext & $:$ & 1305833 \\
\hline \hline
\end{tabular}




\section{Keywords}

\section{Introduction}

Angiogenesis is the remodelling of existing vasculature either by formation of new blood vessels or regression of superfluous vessels. In normal adults there is relatively little angiogenesis, but it is well documented that neovascularisation is required for tumour growth beyond a critical size. The (ER) antagonist TAM is currently the first line therapy for treatment of hormone sensitive breast cancer. However, besides its anti-oestrogenic effects, TAM has also been shown to have anti-angiogenic properties. Although there has been much work on the former, the anti-angiogenic actions of TAM are not well understood.

\section{Aims}

This study set out to explore if the ER antagonist TAM has anti-angiogenic properties in three different in vivo angiogenesis assays.

\section{Comments}

Anti-oestrogens like tamoxifen (TAM) are the treatment of choice for oestrogen receptor (ER) positive, hormone sensitive breast cancers. However, TAM has additional anti-angiogenic effects which are less well documented. Here, the anti-angiogenic properties of TAM were observed in an ER-deplete system. This additional therapeutic effect of anti-oestrogens may explain the beneficial effects which are sometimes observed in ER negative breast tumours. At present it is not known how TAM exerts its antiangiogenic effect. Given that there are now two ERs, it is tempting to speculate that ER ß may have a role in this process. Whatever the mechanism, defining the anti-angiogenic mechanisms of TAM (and other ER antagonists) will be important as it may broaden the use of this already very important drug in the clinic. 


\section{Methods}

Ovariectomised female Fischer 344 rats were treated with either continuous release placebo or TAM pellets $(25 \mathrm{mg})$ for $72 \mathrm{~h}$ prior to the implantation of an ER negative rat fibrosarcoma. Rats were killed 10 days later and the tumours removed for immunohistochemical analysis of microvessel density. In a separate study, aortic rings were prepared from cannulated aortas of ovariectomised female rats and plated in Matrigel 12-well plates. Microvessel sprouting in response to tamoxifen $(0.02 \mathrm{mM}, 0.02 \mu \mathrm{M})$ or vehicle was scored. For the corneal pocket model, rats were randomised into placebo, estradiol (1.5 $\mathrm{mg}$ ) or TAM (25 mg) for $72 \mathrm{~h}$ prior to implantation of a basic fibroblast growth factor pellet into the cornea. After 7 days, the eye was removed and the cornea examined for evidence of vascular sprouting.

\section{Results}

Fibrosarcomas from TAM treated animals had significantly reduced vessel formation compared with controls. None of the tumours had immunohistochemically detectable ER or progesterone receptor. Similarily, TAM inhibited microvessel sprouting in the aortic ring model, irrespective of TAM concentration. In the corneal pocket model, TAM treated animals had significantly decreased vascularity and vessel length in comparison to the control or oestradiol-treated groups.

\section{Discussion}

Using three different animal models, this study confirms that TAM is a potent anti-angiogenic agent. Its action would appear to be independent of ER and suggests a possible contributory mechanism by which TAM reduces the degree of angiogenesis in addition to its well documented anti-oestrogenic role. These dual effects have important implications for breast cancer treatment particularly in those patients with ER negative disease.

\section{References}


1. Blackwell KL, Haroon ZA, Shan S, Saito W, Broadwater G, Greenberg CS, Dewhirst MW:

Tamoxifen inhibits angiogenesis in oestrogen receptor-negative animal models. Clin Cancer Res. 2001, 6: 4359-4364. 